\title{
Isoacceptor specific characterization of tRNA aminoacylation and misacylation in vivo
}

\author{
Kyle Mohler ${ }^{1,3}$, Rebecca Mann ${ }^{2}$ and Michael Ibba ${ }^{1,2,3}$ \\ ${ }^{1}$ Department of Microbiology, ${ }^{2}$ The Ohio State Biochemistry Program, and the ${ }^{3}$ Center for RNA \\ Biology, The Ohio State University, Columbus, Ohio 43210, USA
}

Running title: Isoacceptor Specific Aminoacylation Profiling

Address correspondence to: Michael lbba

Department of Microbiology

The Ohio State University

484 West 12th Ave.

Columbus, Ohio 43210.

Tel. 614-292-2120

Fax. 614-292-8120

E-mail: ibba.1@osu.edu 


\section{Abstract}

Amino acid misincorporation during protein synthesis occurs due to misacylation of tRNAs or defects in decoding at the ribosome. While misincorporation of amino acids has been observed in a variety of contexts, less work has been done to directly assess the extent to which specific tRNAs are misacylated in vivo, and the identity of the misacylated amino acid moiety. Here we describe tRNA isoacceptor specific aminoacylation profiling (ISAP), a method to identify and quantify the amino acids attached to a tRNA species in vivo. ISAP allows compilation of aminoacylation profiles for specific isoacceptors tRNAs. To demonstrate the efficacy and broad applicability of ISAP, $\operatorname{tRNA}^{\text {Phe }}$ and $\operatorname{tRNA}^{\mathrm{Tyr}}$ species were isolated from total aminoacyl-tRNA extracted from both yeast and Escherichia coli. Isolated aminoacyl-tRNAs were washed until free of detectable unbound amino acid and subsequently deacylated. Free amino acids from the deacylated fraction were then identified and quantified by mass spectrometry. Using ISAP allowed quantification of the effects of quality control on the accumulation of misacylated tRNA species under different growth conditions. 


\section{Introduction}

tRNA is the most ubiquitous small non-coding RNA in the cell, comprising $10-14 \%$ of the total intracellular RNA pool [1]. As direct mediators of translational fidelity, aminoacyl-tRNA synthetases (aaRS) are responsible for the correct pairing of an amino acid with its cognate tRNA [2]. Phenylalanyl-tRNA synthetase (PheRS), for example, is responsible for pairing phenylalanine (Phe) with tRNA ${ }^{\text {Phe }}$ [3]. Mispaired aminoacyl-tRNA (aa-tRNA) species occasionally arise due to a lack of adequate amino acid discrimination within the PheRS active site, resulting in the synthesis of misacylated Tyr-tRNA ${ }^{\text {Phe }}[4]$. The ability of PheRS to misacylate tRNA ${ }^{\text {Phe }}$ with non-cognate amino acid makes tRNA proofreading mechanisms of PheRS essential to maintaining the accuracy of translation $[3,5]$.

While misincorporation of amino acids has been observed in a variety of contexts, less work has been done to directly assess the extent to which specific tRNAs are misacylated in vivo or to identify the misacylated amino acid moiety. Characterization of amino acid misacylation in vitro using recombinantly expressed aaRSs is a powerful tool for determining the kinetic parameters for specific amino acid/tRNA pairs, however this approach presupposes that the identity of the amino acid is known in advance. In order to directly determine the identity of amino acids misacylated to specific tRNAs, we have developed a tRNA pulldown method which allows determination of global aminoacylation profile for a given tRNA isoacceptor. We have extensively validated the efficacy of our method using radiolabeled amino acids aminoacylated to cognate and non-cognate tRNAs. Using this approach, we have observed high sequence specificity within I tRNA isoacceptor groups with sufficient divergence of primary sequence and minimal cross reactivity with off target tRNAs.

\section{Description of Method}


Small RNAs are selectively isolated under acidic conditions to maintain the labile ester linkage between tRNAs and the attached amino acids, which is prone to nucleophilic attack in the presence of free hydroxyl radicals Individual aa-tRNAs are then isolated by hybridization with tRNA isoacceptor specific biotinylated DNA oligonucleotides. Hybridized aa-tRNA/DNA are then captured with streptavidin conjugated para-magnetic particles (SA-PMPs). The streptavidinbiotin is one of the highest affinity interactions found in nature making it ideal for the efficient capture of biomolecules. Magnets are then used to effectively separate the biomolecule conjugated SA-PMPs from the non-target aa-tRNAs in the supernatant. The SA-PMPs are washed extensively to remove free amino acids and non-target aa-tRNAs under acidic conditions to prevent deacylation. Following removal of contaminating aa-tRNAs, the SA-PMPs are incubated under basic conditions in which free hydroxyl groups attack the ester linkage at the terminal $3{ }^{\prime} \mathrm{OH}$ ribose sugar, thus freeing the amino acid moiety from the bound tRNA. The tRNA/SA-PMPs are removed from the deacylation reaction, leaving the amino acids behind for further analysis (Fig. 1).

\section{Experimental procedures}

\subsection{Isolation of Small RNA under Acidic Conditions}

This method is suitable for the isolation of small RNAs from both Escherichia coli and Saccharomyces cerevisiae. The yield of small RNA from liquid culture is typically $1 \mu \mathrm{g}$ per $\mathrm{mL}$. The amount of culture used should be scaled according to the intended application (discussed in Applications). Standard precautions to avoid RNase contamination should be observed where possible.

- Growth conditions and volumes for cell cultures will vary with experimental design. Once cells reach the desired density, immediately chill cultures on ice. Cells are harvested by 
centrifugation at $4{ }^{\circ} \mathrm{C}$ (Yeast: 5 minutes at $2000 \mathrm{xg}$, E. coli: 5 minutes at $3000 \mathrm{rpm}$ ) and the supernatant is removed.

- Cells are then re-suspended in $400 \mu$ of ice-cold aqueous phase lysis buffer and transferred to a $2 \mathrm{~mL}$ screw cap microcentrifuge tube. Lysis buffer should be freshly prepared using RNase free components. Prepare the aqueous phase of the lysis buffer by making a solution of $300 \mathrm{mM} \mathrm{NaOAc} \mathrm{pH} 4.5$ and 10mM EDTA. Add an equal volume of phenol/ $\mathrm{CHCl}_{3} \mathrm{pH} 4.5$ to the aqueous phase lysis buffer cell suspension. Vortex the cell suspension for 30 seconds. Repeat 3-6 times, chilling on ice for at least 60 seconds in between each round.

- Separate the aqueous phase of the lysis buffer from the organic phase by centrifugation at $4{ }^{\circ} \mathrm{C}$ for 15 minutes at $18,600 \mathrm{xg}$. Remove the aqueous phase (containing the RNA) to a fresh RNase free microcentrifuge tube. Repeat the phenol/ $\mathrm{CHCl}_{3}$ extraction by adding an equal volume of phenol/ $\mathrm{CHCl}_{3} \mathrm{pH} 4.5$ to the newly extracted aqueous phase. Separate the aqueous phase as described above and remove to a fresh RNase free microcentrifuge tube.

- Precipitate the RNA from the aqueous phase by adding $2.7 x$ the volume of $100 \%$ RNase free ethanol and chilling at $-20^{\circ} \mathrm{C}$ for 1 hour or $-80^{\circ} \mathrm{C}$ for 30 minutes. Pellet the nucleic acids by centrifugation at $4{ }^{\circ} \mathrm{C}$ for 15 minutes at $18,600 \mathrm{xg}$ and discard the supernatant. Re-dissolve the pellet in aqueous phase lysis buffer and repeat precipitation of the nucleic acids as previously described.

- Allow the nucleic acid pellet to dry slightly. Do not over dry the pellet. Re-suspend the pellet in $100 \mathrm{mM} \mathrm{NaOAc} \mathrm{pH}$ 4.5. Analyze the purity and quantity of the RNA by 
spectrophotometry, fluorimetry or gel electrophoresis. The bioanalyzer trace (Fig. 2) depicts a typical result from this purification protocol. Use RNA immediately or store RNA at $-80^{\circ} \mathrm{C}$ for up to two weeks.

\subsection{Isoacceptor Specific tRNA Probes}

\subsubsection{Probe Design}

Proper probe design is essential to the success of ISAP. The tRNA pool comprises the most highly modified RNA population within the cell. Post-transcriptional modification of RNAs are essential, providing structural and functional tuning (Fig. 3). Therefore, when designing tRNA specific probes, care must be taken to avoid overlap with nucleotides possessing posttranscriptional modifications which inhibit canonical Watson-Crick base pairing. Base methylation, for example, has been shown to destabilize hybridization of the entire probe. In addition to considering base modifications, the probe sequence must be carefully chosen to minimize the possibility of off-target capture. The length of the probe can be varied between 2040 nucleotides depending upon GC content of the target sequence.

\subsection{Targeted capture of aa-tRNA}

\subsubsection{Probe hybridization}

After isolating RNA as described above, the aa-tRNA pool may be analyzed using probes designed to target a specific tRNA isoacceptors. If hybridization conditions for the target tRNA have not been previously established, optimization of hybridization may be necessary to maximize capture while minimizing deacylation of the aa-tRNA and cross-reactivity.

- In an RNase free microcentrifuge tube, combine total RNA (amount will vary depending upon intended application) with $1 \mu \mathrm{M} 3$ ' biotinylated probe in $500 \mu \mathrm{l}$ of $2 \mathrm{XSSC}, \mathrm{pH} 4.8$. Incubate the RNA/Probe mixture for 1 hour at $50 \mathrm{C}$. 
- Remove the RNA/probe mixture from heat and allow the mixture to cool to room temperature.

\subsubsection{Probe capture}

- While the mixture is cooling, pre-wash $0.5 \mathrm{mg}$ of streptavidin conjugated paramagnetic particles (SA-PMPs). Re-suspend the SA-PMPs in the storage buffer by gently mixing the bottle. Pipet the volume equivalent to $0.5 \mathrm{mg}$ of SA-PMPs to a fresh RNase free microcentrifuge tube. The SA-PMPs may be captured using a magnetic separation rack or, as used in this protocol, a single channel magnetic pipet. Capture the SA-PMPs using a magnetic pipet and transfer them to a new microcentrifuge tube pre-filled with $300 \mu \mathrm{l}$ of $2 \mathrm{X} \mathrm{SSC}, \mathrm{pH}$ 4.8. Repeat this procedure to wash the SA-PMPs a total of 3 times in $2 \mathrm{X} \mathrm{SSC}, \mathrm{pH}$ 4.8. Following the final wash, move the SA-PMPs to a new microcentrifuge tube pre-filled with $100 \mu \mathrm{l}$ of $2 \mathrm{XSSC}, \mathrm{pH} 4.8$.

- After the RNA/probe mixture has cooled to room temperature, capture the annealed oligonucleotides by adding the $500 \mu \mathrm{l}$ RNA/probe mixture to the $100 \mu \mathrm{l}$ of pre-washed SA-PMPs. Incubate the capture reaction at room temperature for 10 minutes. Gently mix the tube by inverting every 1-2 minutes.

\subsubsection{Amino acid Isolation}

The captured RNA-probe mixture is highly contaminated with off-target RNA and unhybridized probe and must be extensively washed to remove contaminating aa-tRNA.

- Using a magnetic pipet, capture the SA-PMPs and move them to a new microcentrifuge tube pre-filled with $300 \mu \mathrm{l}$ of $2 \mathrm{XSSC}, \mathrm{pH} 4.8$. Repeat this wash step for a total of 6 
washes. Be sure to move the SA-PMPs bound with the target aa-tRNA to a new tube for each wash.

- After washing the SA-PMPs free from contaminating nucleic acids, the amino acid moieties must be removed from the aminoacyl-tRNA bound to the SA-PMPs. Deacylate the tRNA by moving the SA-PMPs to a new microcentrifuge tube pre-filled with $200 \mu$ of $20 \mathrm{mM}$ ammonium formate, $\mathrm{pH} 10$. Incubate the deacylation reaction at $37^{\circ} \mathrm{C}$ for 1 hour. After the deacylation reaction is complete, remove the SA-PMPs using a magnetic pipet. The amino acids previously bound to the tRNAs are now in the supernatant.

\subsubsection{Validation of Probe Efficacy and Specificity}

Proper probe design can dramatically reduce the interaction of a probe with non-target tRNAs. However, when using a new probe, it is important to validate the efficacy and specificity of the probe towards the target tRNA. To test probe efficacy, radiolabeled aa-tRNA may be prepared in vitro and used to monitor the status of aa-tRNA throughout the ISAP protocol. Additionally, radiolabeled amino acids may be quantified using liquid scintillation to assess amino acid recovery following deacylation of aa-tRNA. Radiolabeled aa-tRNA may be pre-formed using in vitro transcribed tRNA corresponding to the target tRNA. As modifications may alter probe hybridization, the use of total modified tRNA from the target organism should be preferred. S100 extract may be prepared from the target organism to provide the pool of aminoacyl-tRNA synthetases necessary for the aminoacylation reaction. Probe hybridization should be assayed against target and non-target aa-tRNA. Properly designed probes should recover $>50 \%$ of aatRNA, while failing to capture radiolabeled non-target tRNA species (Fig. 4A). Complete recovery of target aa-tRNA is limited by deacylation during hybridization or failure to efficiently capture all target aa-tRNAs. As the stability of the ester linkage is largely mediated by the identity of the amino acid moiety, care must be taken to optimize hybridization temperatures to 
maximize recovery while minimizing temperature mediated hydrolysis of target aa-tRNAs [6]. Probe specificity may be examined using standard northern blot techniques for tRNAs. Probe hybridization should yield a single band when deacylated and two bands when using acid-urea gel electrophoresis to separate aa-tRNA from deacylated tRNA (Fig. 4B). Validation of tRNA probes is essential to the interpretation of results, especially when high sensitivity detection of amino acids is required.

\section{Identification and quantification of amino acids}

Analysis of amino acids should be conducted following deacylation of captured tRNA. Numerous detection assays may be employed depending upon the experimental goals. For the sensitive detection of amino acids, particularly when looking for a small population of noncognate amino acids, analysis using mass spectrometry is a powerful approach. However, care must be taken when the identity of the population of amino acids aminoacylated to a tRNA is unknown. Additionally, specific protocols must be designed to evaluate the presence of nonproteogenic amino acids, or amino acid derivatives [7].

\section{Applications}

\subsection{Identification of aminoacyl moieties}

In certain organisms, the identity of the tRNA is difficult to determine based on primary tDNA gene sequence. tRNA modification patterns can also alter the recognition of tRNA substrates by aaRSs, resulting in altered aminoacylation specificity [8]. In these situations, ISAP is an effective tool for the identification of amino acid moieties aminoacylated to an individual tRNA isoacceptor. As an example, a tRNA probe for $\operatorname{tRNA}^{\text {Phe }}$ from $S$. cerevisiae was used to capture the target tRNA and identify the amino acid moiety. Mass spectrometry analysis of the amino acids deacylated from the target tRNA identified the major amino acid population as Phe, corresponding to the target tRNA (Fig. 5). 


\subsection{Detection of misacylated tRNA}

In some pathogenic organism, the misacylation of tRNAs plays a central role in virulence and adaptation to host immune response [8]. ISAP presents a novel method for the identification and quantification of in vivo misacylated tRNAs. In addition to the identification of cognate amino acid moieties for a target tRNA, the identity and relative quantity of misacylated tRNA may be assayed with variable sensitivity depending upon the amino acid analysis method. For example, using total RNA extracted from WT and PheRS quality control (editing) deficient strains of yeast, aa-tRNA ${ }^{\text {Phe }}$ can be selectively isolated and the amounts of misacylated Tyr relative to levels of cognate Phe determined (Fig. 6)

\subsection{Biomarker discovery}

The use of tRNAs as biomarkers for disease models is relatively unexplored compared to other small RNAs. In the tumor microenvironment, cells are often starved for amino acid substrates. Amino acid limitation can disrupt the amino acid pool, altering the selectivity of aaRSs and leading to the misacylation of tRNA. Misacylated tRNA substrates can be used to maintain high translation rates while simultaneously increasing the yield of mistranslated proteins within the cell. ISAP is uniquely positioned to explore the profile of misacylated tRNAs within the cell. Profiling the tRNA pool within the context of human disease is likely to yield novel targets for therapeutic investigation.

\section{Concluding remarks}

tRNAs are important biomolecules in the context of human disease. Numerous pathologies result from defects in the aa-tRNA proofreading domains of aaRSs. Historically, aa-tRNA proofreading defects have been characterized by their ability to generate misacylated tRNA in vitro. However, the use of ISAP allows for the characterization of the impact of aaRS

proofreading defects on aa-tRNA pool fidelity within the biological context of the organism. 
Although only a few applications are listed here, the ability of ISAP to further our understanding of the biological roles that tRNAs misacylated with both canonical and non-proteinogenic amino acids play within the cell is virtually unlimited.

\section{Acknowledgements}

This work was supported by grants from the National Science Foundation (MCB 1412611) and the Army Research Office (W911NF1510105), NIH Training Grant T32 GM086252 (K.M.) and a fellowship from the OSU Center for RNA Biology (K.M.).

\section{References}

[1] S.S. Grewal, Why should cancer biologists care about tRNAs? tRNA synthesis, mRNA translation and the control of growth, Biochimica et biophysica acta 1849(7) (2015) 898-907.

[2] S.S. Yadavalli, M. Ibba, Quality control in aminoacyl-tRNA synthesis its role in translational fidelity, Adv. Protein Chem. Struct. Biol. 86 (2012) 1-43.

[3] L. Klipcan, I. Finarov, N. Moor, M.G. Safro, Structural Aspects of Phenylalanylation and Quality Control in Three Major Forms of Phenylalanyl-tRNA Synthetase, Journal of amino acids 2010 (2010) 983503.

[4] J. Ling, S.S. Yadavalli, M. Ibba, Phenylalanyl-tRNA synthetase editing defects result in efficient mistranslation of phenylalanine codons as tyrosine, RNA 13(11) (2007) 1881-1886.

[5] H. Roy, J. Ling, J. Alfonzo, M. Ibba, Loss of editing activity during the evolution of mitochondrial phenylalanyl-tRNA synthetase, J. Biol. Chem. 280 (2005) 38186-38192.

[6] J.R. Peacock, R.R. Walvoord, A.Y. Chang, M.C. Kozlowski, H. Gamper, Y.M. Hou, Amino acid-dependent stability of the acyl linkage in aminoacyl-tRNA, Rna 20(6) (2014) 758-64.

[7] T.J. Bullwinkle, N.M. Reynolds, M. Raina, A. Moghal, E. Matsa, A. Rajkovic, H. Kayadibi, F. Fazlollahi, C. Ryan, N. Howitz, K.F. Faull, B.A. Lazazzera, M. Ibba, Oxidation of cellular amino acid pools leads to cytotoxic mistranslation of the genetic code, eLife 3 (2014) 02501.

[8] T. Muramatsu, K. Nishikawa, F. Nemoto, Y. Kuchino, S. Nishimura, T. Miyazawa, S. Yokoyama, Codon and amino-acid specificities of a transfer RNA are both converted by a single post-transcriptional modification, Nature 336(6195) (1988) 179-81. 


\section{Figure legends}

Figure 1: Graphical depiction of ISAP workflow. tRNA is isolated and hybridized to a biotinylated oligonucleotide probe. The probe is captured and the amino acid moiety is isolated and analyzed.

Figure 2: Bioanalyzer electropherogram data for purified RNA. Yeast RNA was extracted following the protocol outlined in 4.1. Populations of small RNAs, including tRNAs and 5S RNA, are preferentially isolated under acidic conditions.

Figure 3: Global modifications of tRNA from E. coli and S. cerevisiae. Post transcriptional modifications of tRNA bases are compiled for all tRNAs in E. coli and S. cerevisiae to demonstrate the level of modification at each position within the tRNA. tRNA modifications compiled and displayed using Modomics.

Figure 4: Probe efficacy and specificity. tRNAs from E. coli were aminoacylated with radiolabeled amino acids and used for in vitro ISAP optimization. A: E. coli ${ }^{14} \mathrm{C}-\mathrm{Phe}$-tRNA ${ }^{\text {Phe }}$ and ${ }^{14} \mathrm{C}-\mathrm{Tyr}$-tRNA ${ }^{\mathrm{Tyr}}$ were prepared in vitro and used as substrates for ISAP probe characterization. Probes for tRNA ${ }^{\text {Phe }}$ (5'-CCGGACTCGGAATCGAACCAAGGACACGGGG[BtnTg]-3') and tRNA $^{\text {Tyr }}$ (5'-GGGAAGGATTCGAACCTTCGAAG[BtnTg]-3') were used to attempt capture of target and non-target tRNAs. B: tRNA northern blot using tRNA ${ }^{\text {Phe }}$ ISAP probe specific for yeast tRNA $^{\text {Phe }}$ (5'BTN-GATCGAACACAGGACCTCCAGAT-3').

Figure 5: Amino acids analysis of yeast aa-tRNA. aa-tRNAPhe from $S$. cerevisiae was isolated and captured using a probe specific for tRNA ${ }^{\text {Phe }}$. Amino acid identification and relative quantification was conducted using mass spectrometry. 
Figure 6: Profiling of misacylated tRNAs. aa-tRNA ${ }^{\text {Phe }}$ from $S$. cerevisiae was isolated and captured using a probe specific for tRNA ${ }^{\text {Phe }}$. Relative quantities of non-cognate amino acids were determined using mass spectrometry analysis. 


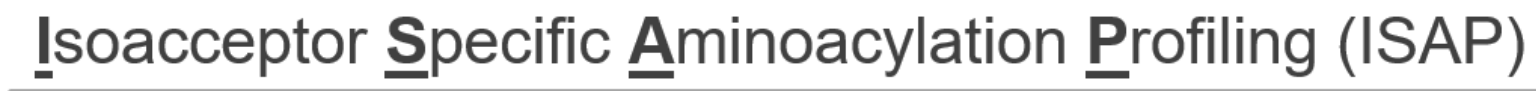

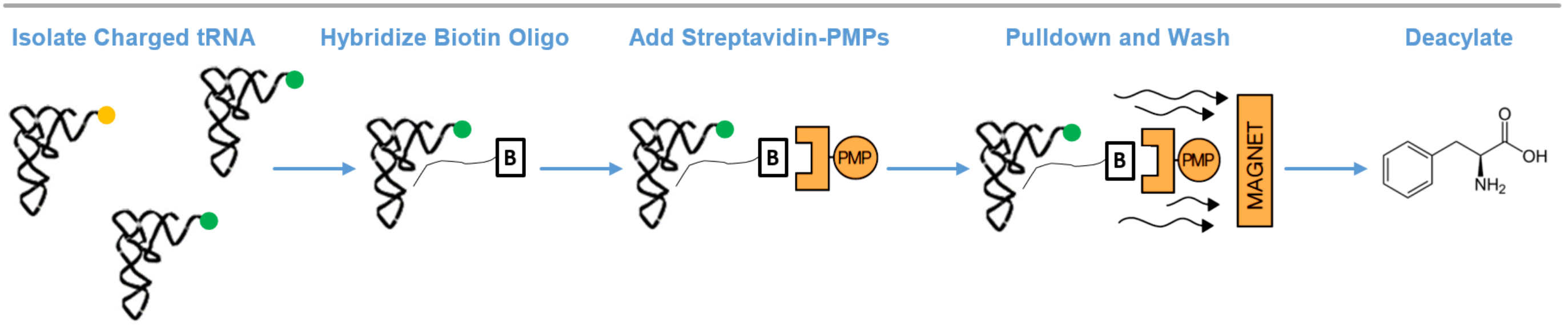


S. cerevisiae

3'

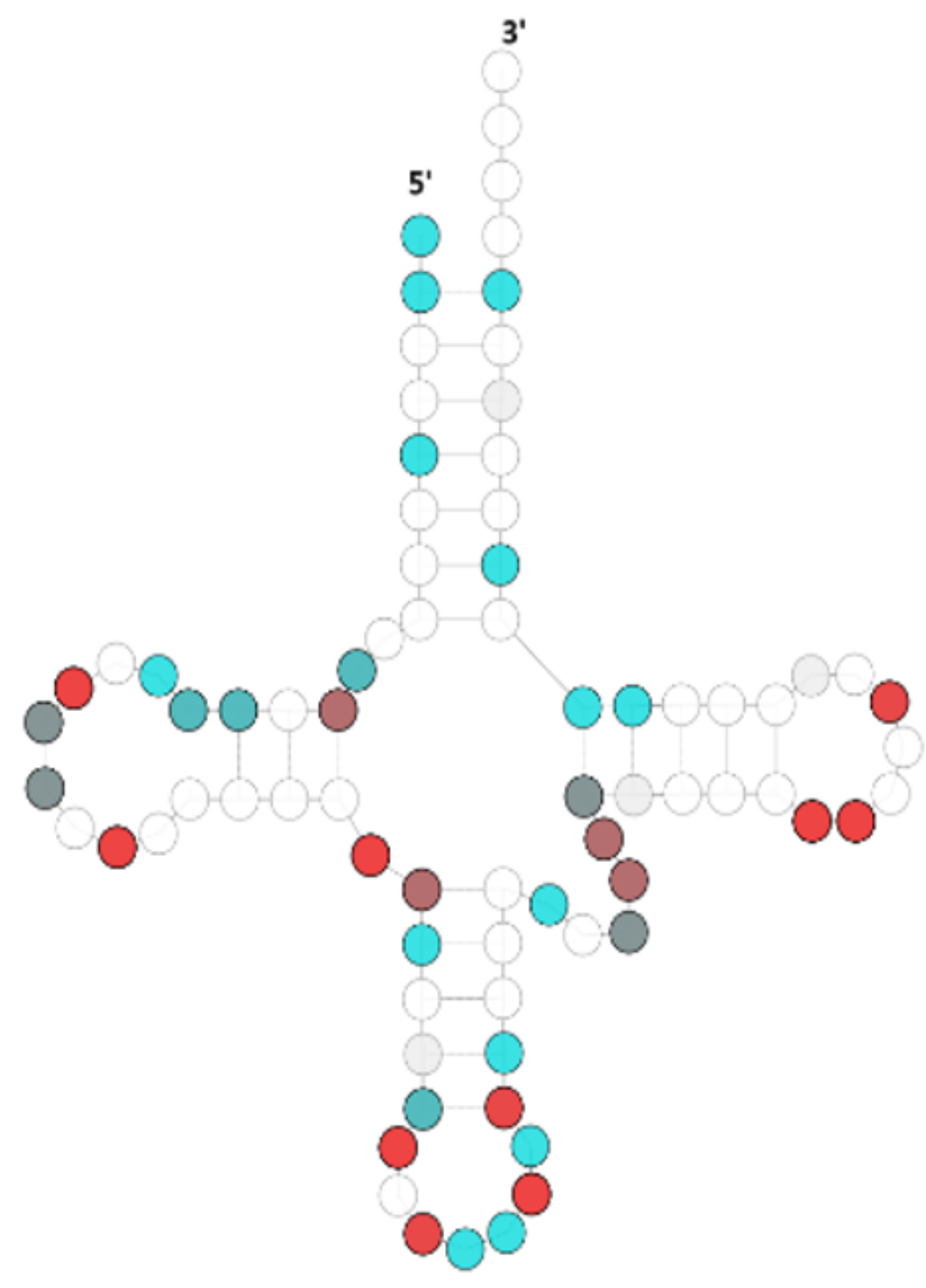

E. coli

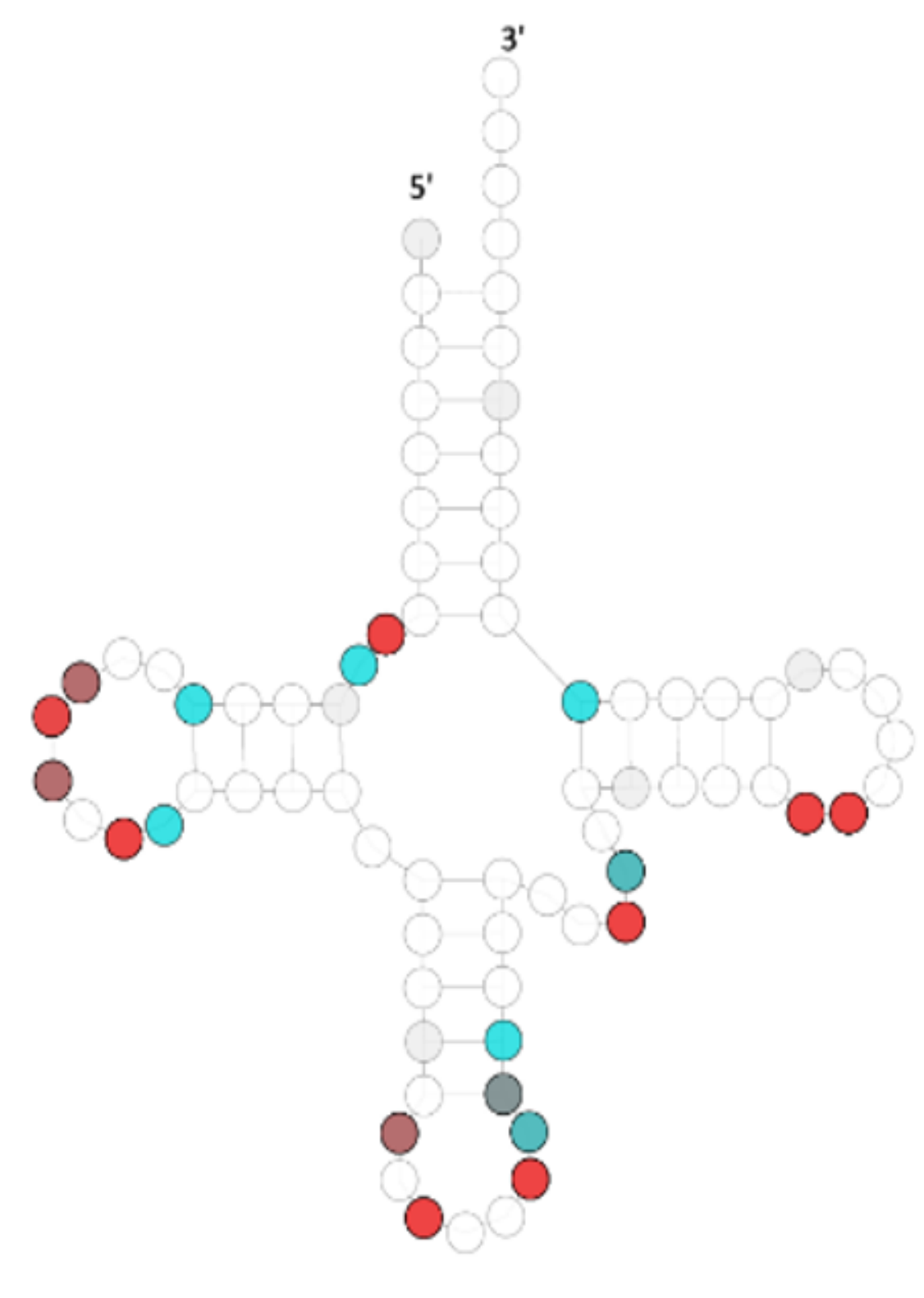

modification scale: up to $20 \% \quad 40 \% \quad 60 \%$ 


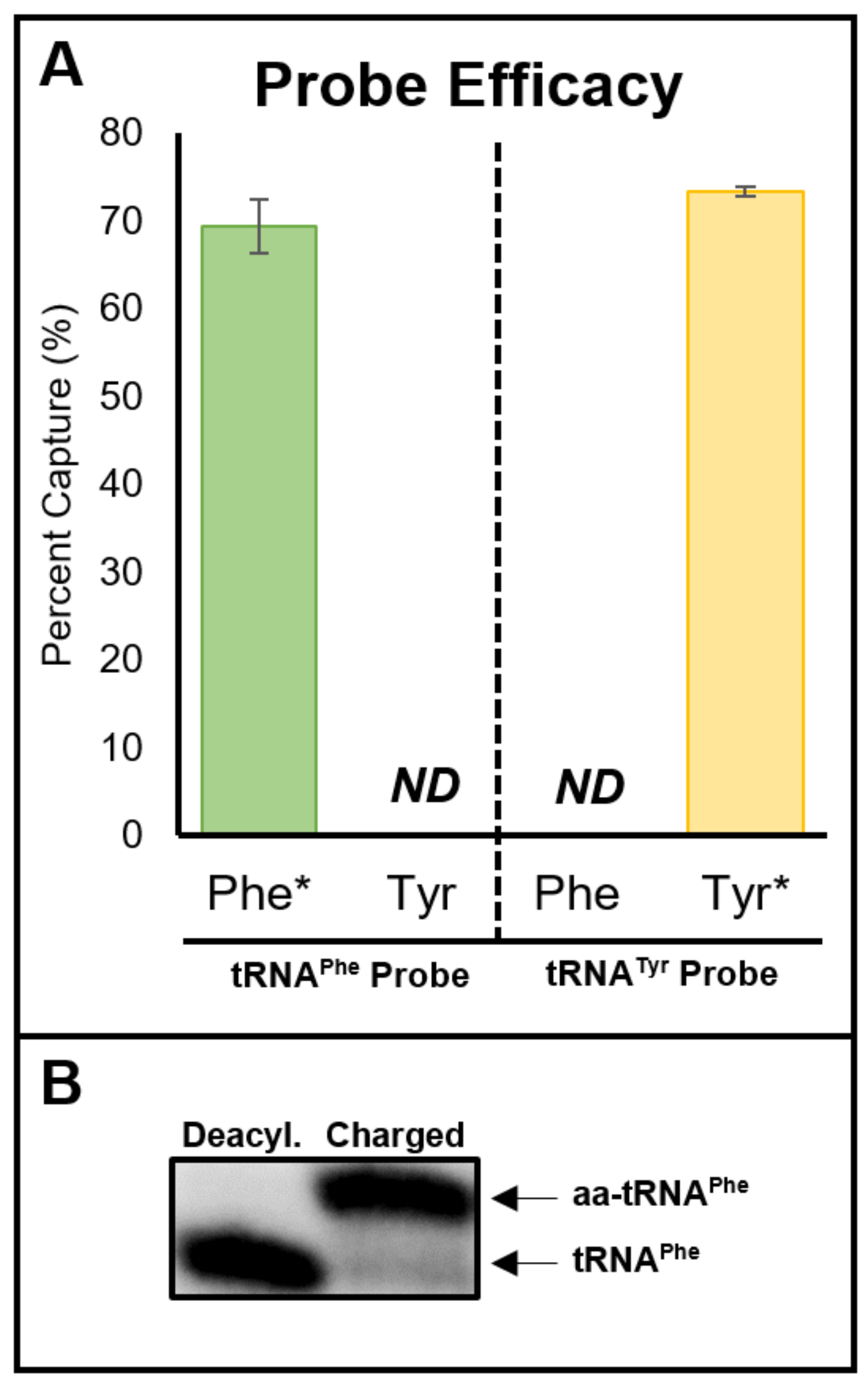

Figure 4 
